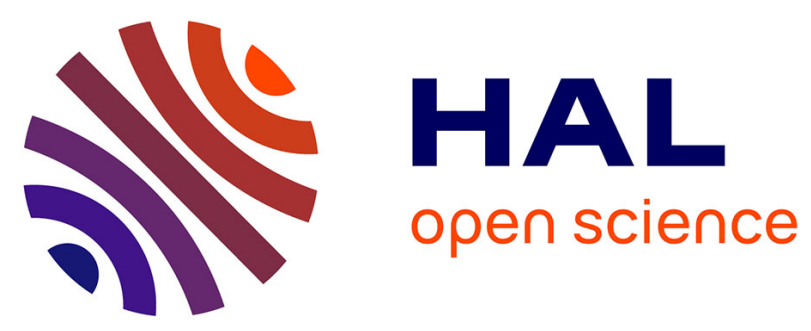

\title{
La spectrométrie de fluorescence $X$ pour l'analyse quantitative des végétaux et de leurs produits résiduels de combustion
}

S. Garivait, J. Quisefit, E. Steiner, P. de Châteaubourg

\section{- To cite this version:}

S. Garivait, J. Quisefit, E. Steiner, P. de Châteaubourg. La spectrométrie de fluorescence X pour l'analyse quantitative des végétaux et de leurs produits résiduels de combustion. Journal de Physique IV Proceedings, 1996, 06 (C4), pp.C4-823-C4-832. 10.1051/jp4:1996479 . jpa-00254362

\section{HAL Id: jpa-00254362 https://hal.science/jpa-00254362}

Submitted on 1 Jan 1996

HAL is a multi-disciplinary open access archive for the deposit and dissemination of scientific research documents, whether they are published or not. The documents may come from teaching and research institutions in France or abroad, or from public or private research centers.
L'archive ouverte pluridisciplinaire HAL, est destinée au dépôt et à la diffusion de documents scientifiques de niveau recherche, publiés ou non, émanant des établissements d'enseignement et de recherche français ou étrangers, des laboratoires publics ou privés. 


\title{
La spectrométrie de fluorescence $X$ pour l'analyse quantitative des végétaux et de leurs produits résiduels de combustion
}

\author{
S. Garivait, J.P. Quisefit, E. Steiner et P. de Châteaubourg \\ Laboratoire Interuniversitaire des Systèmes Atmosphériques (LISA), Universités Paris 7-Paris 12, \\ URA 1404 du CNRS, 61 avenue du Général de Gaulle, 94010 Créteil cedex, France
}

\begin{abstract}
Résumé : Afin d'établir un bilan de matière des émissions gazeuses et particulaires des feux de savane, une des principales sources de composés atmosphériques, nous avons été amenés à quantifier la composition chimique élémentaire des végétaux combustibles et des cendres de combustion. Pour ce faire, deux principales techniques ont été utilisées : la Microanalyse (MA) pour caractériser la fraction organique et la Spectrométrie de Fluorescence X (SFX) pour la fraction minérale. Afin de couvrir toute la gamme d'éléments recherchés par la SFX ( $\mathrm{Si}, \mathrm{P}, \mathrm{Mg}$, $\mathrm{Fe}, \mathrm{Mn}, \mathrm{K}, \mathrm{Ca}, \mathrm{Na}, \mathrm{S}$ et $\mathrm{Cl}$ ), nous avons mis en oeuvre plusieurs protocoles complémentaires de préparation d'échantillon : fabrication des perles à partir des poudres végétales et des cendres, préparation des échantillons couches-minces (filtres) à partir des cendres préalablement mises en suspension dans un liquide inerte, et mise en cellule des poudres végétales pour leur analyse directe suivant la méthode de correction utilisant la mesure des intensités diffusées.
\end{abstract}

\begin{abstract}
In order to establish the chemical balance of gases and particles emitted by savannah grasses burning, one of the major atmospheric compounds sources, we have due to determine the elementary composition of biomass fuel and its combustion ashes. In this aim, we have used two main analytical techniques : Microanalysis (MA) for the determination of the organic fraction and X-rays Fluorescence Spectrometry (XRF) for the mineral one. To cover the whole range of elements of interest with XRF ( $\mathrm{Si}, \mathrm{P}, \mathrm{Mg}, \mathrm{Fe}, \mathrm{Mn}, \mathrm{K}, \mathrm{Ca}, \mathrm{Na}, \mathrm{S}$ and $\mathrm{Cl}$ ), we carried out complementary procedures of samples preparation : glass-disc setting of plants and ashes, thin-film preparation from ashes preliminary suspended in an inert liquid, and cell-filled of plants powder for its direct analysis by the method using scattered radiation correction.
\end{abstract}

\section{INTRODUCTION}

La combustion de biomasse végétale est principalement liée à la pratique des feux de forêt et de savane, en particulier dans les régions tropicale et subtropicale [1]. Lorsqu'un végétal est brûlé, il se produit d'une part des émissions gazeuses et particulaires et d'autre part des résidus de combustion ou cendres. Compte tenu de l'intensité de leurs flux d'émission et de la quantité de végétal brûlé chaque année, les feux de savane constituent une des sources majeures de composés atmosphériques [2]. Les différentes études de terrain ou en laboratoire 
ont montré que les émissions des feux de biomasse végétale sont qualitativement influencées par deux principaux facteurs [1-4] :

1/ les modes majeurs de combustion observables au cours d'un feu : l'une appelée "flaming" produisant des flammes et l'autre "smoldering" n'émettant que des fumerolles blanchâtres .

2/ la composition des végétaux combustibles : par exemple, le marquage en $\mathrm{K}, \mathrm{S}$ ou $\mathrm{Cl}$, des particules émises n'a pu être expliqué que par la présence de ces éléments dans les plantes à brûler $[3,5]$.

Afin d'établir le bilan de matière des émissions des feux de savane, nous avons donc été amené à quantifier linfluence de ces deux facteurs sur la formation des produits de combustion. Pour ce faire, nous avons effectué une série d'expériences spécifiques de feux de savane en chambre de combustion. Cette expérimentation, partie intégrante du programme CNRSDECAFE $^{1}$, a été réalisé au Centre de Recherche Atmosphérique (CRA) à Lannemezan (France) et a réuni plusieurs laboratoires spécialisés dans l'étude des systèmes atmosphériques : LISA $^{2}$, LA $^{3}$, LESAM $^{4}$, CRA $^{5}$ et CFR $^{6}$. Pour chaque expérience, nous avons défini le végétal combustible et le mode de combustion, puis échantillonné les produits formés correspondants.

Pour caractériser la composition élémentaire des végétaux et des cendres, peu documentée jusqu'à présent [6], nous avons utilisé deux principales techniques : la Microanalyse (MA) pour l'analyse du carbone, de l'hydrogène, de l'azote et de l'oxygène organique ; et la Spectrométrie de Fluorescence $X$ ( $\mathrm{SFX}$ ) pour les éléments minéraux d'intérêt (K, $\mathrm{Ca}, \mathrm{Si}, \mathrm{P}, \mathrm{Na}, \mathrm{Mg}, \mathrm{Fe}, \mathrm{Mn}$, $\mathrm{S}$ et $\mathbf{C l}$ ).

Dans cet article, nous décrivons l'analyse par SFX que nous avons développé avec un appareil dispersif en longueur d'onde (Siemens SRS 303). La partie principale de ce développement a été la mise au point des procédures complémentaires de préparation d'échantillon adaptées à l'analyse par SFX et qui permettent de documenter l'ensemble des éléments recherchés.

\section{PROCEDURE D'ANALYSE DES VEGETAUX :}

Les herbes de savane utilisées ont été collectées durant les expériences des feux de terrain FOS $^{7} / \mathrm{DECAFE}$ à Lamto (Côte d'Ivoire) et SAFARI ${ }^{8}$ à Parc Kruger (Afrique du Sud). Les végétaux de Lamto ont été ideutifiées comme étant du Loudetia mélangé ou des Andropogons, alors que ceux de l'Afrique du Sud sont un mélange de graminées que nous nommerons SAFARI. Ces herbes de savane sont composés à plus de $80 \%$ de cellulose [4]. Les teneurs des minéraux dans ce type de végétal ont été peu documentées : seules celles des éléments nutritifs tels que $\mathrm{K}$ et $\mathbf{P}$ ont été étudiées [7]. Pour analyser la fraction minérale contenue dans une matrice végétale, la technique généralement utilisée est la Spectrométrie d'Absorption Atomique (SAA) [8,9]. Celle-ci nécessite au préalable une mise en solution de l'échantillon végétal, et donc une destruction totale de la matrice d'origine. Ceci s'effectue soit par une décomposition thermique ou par laser, soit par une minéralisation [8-12]. Ces méthodes peuvent conduire à une perte en certains éléments liée à leur insolubilisation et/ou volatilisation. En effet, elles dépendent fortement de la composition chimique de léchantillon

\footnotetext{
${ }^{1}$ DECAFE : Dynamique Et Chimie de l'Atmosphère en Forêt Equatoriale.

${ }^{2}$ LISA : Laboratoire Interuniversitaire des Systèmes Atmosphériques.

${ }^{3}$ LA : Laboratoire d'Aérologie.

${ }^{4}$ LESAM : Laboratoire d'Etude des Systèmes Atmosphériques et Multiphasiques.

"CRA : Centre de Recherche Atmosphérique.

${ }^{6}$ CFR : Centre des Faibles Radioactivités.

${ }^{7}$ FOS : Fos Of Savannah.

${ }^{8}$ SAFARI : South African Fires-Atmospheric Research Initiative.
} 
initial ainsi que de lélément à analyser. Par exemple, il est bien connu que la silice est difficilement soluble sans la présence de l'acide fluoridrique dans la solution d'attaque. De plus, le silicium solubilisé se trouve alors sous forme de composé volatil $\mathrm{SiF}_{6}$.

Pour ces raisons, nous avons préféré déterminier les teneurs des minéraux de nos herbes par SFX. L'avantage de cette technique réside en une analyse directe et non destructive des échantillons solides (sols, roches, aciers, etc.). Néanmoins, il est nécessaire de tenir compte des effets de matrice (interéléments et préparation) perturbant la relation "Intensité MesuréeConcentration". Pour l'analyse des végétaux, nous avons donc appliqué la méthode de correction basée sur la mesure de lintensité diffusée par les différents échantillons analysés [13].

Par ailleurs, l'analyse des mineurs ou traces des solides par SFX nécessite en général une simple préparation de l'échantillon. Un pastillage suffit pour l'échantillon initialement présenté sous forme de poudre $[14,15]$. L'obtention d'une pastille d'épaisseur infinie pour l'élément à analyser constitue une condition indispensable.

Cependant, la SFX demeure peu utilisée pour la détermination de la fraction minérale des végétaux. Ceci est principalement dû à la consommation élevée en poudre végétale pour obtenir une pastille d'épaisseur infinie. En effet, pour l'analyse des différents éléments dont le numéro atomique est supérieur à 10 , il faut plus de $500 \mathrm{mg}$ de poudre végétale pour obtenir une pastille d'épaisseur infinie de $3,2 \mathrm{~cm}$ de diamètre (un diamètre classiquement adapté à celui des passeurs d'échantillon) pressée sous 15 tonnes. Par exemple, $2500 \mathrm{mg}$ sont nécessaires pour déterminer la concentration du zinc par la mesure de $\mathrm{Zn} \mathrm{K}_{\alpha}$ [15].

Un pastillage nécessite par ailleurs un ajout de liant (graphite, cellulose, alcool de polyvinyle, cire, acide borique, etc.) [14-16]. Les essais menés sur les poudres des végétaux disponibles ont montré que la quantité de liant à ajouter dépend de la nature de la matière initiale : herbes, feuilles, tiges etc. Dans le cas des herbes de savane, il faut ajouter au moins 150 à $250 \mathrm{mg}$ de cellulose aux $500 \mathrm{mg}$ de poudre végétale pour préparer une pastille $(3,2 \mathrm{~cm}$ de diamètre, sous 15 tonne) d'épaisseur infinie, pour effectuer l'analyse de l'ensemble des éléments recherchés. La quantité de liant représente donc plus de $20 \%$ de la masse totale de la pastille finale. Dès lors, la composante ajoutée ne joue pas simplement un rôle de liant mais également de diluant. Cet important ajout peut également augmenter considérablement les effets diffusants de l'échantillon à analyser puisque la matrice des liants généralement utilisés est constituée par des éléments légers.

Ainsi, nous avons préféré une préparation qui consiste à présenter l'échantillon sous forme d'une cellule remplie de végétal finement broyé. La cellule employée est en polycarbonate à fenêtre de milar ( $6 \mu \mathrm{m}$ d'épaisseur). Elle est fermée par un filtre en cellulose type Wathman 541 permettant d'éviter les pertes de matière lors de la mise sous vide du spectromètre, condition indispensable pour l'analyse des éléments légers. L'avantage de cette méthode par rapport à un pastillage repose sur son respect total de l'échantillon d'origine. En effet, on est soumis ni à une contamination, ni à une dilution, ni à une perte de certains éléments, liées à la présence d'un tiers corps ou à la destruction de la matrice initiale. Néanmoins, il est nécessaire de tenir compte des effets de grains pouvant affecter la précision analytique. Les résultats obtenus ont montré que la méthode de correction basée sur les intensités diffusées semble permettre également de corriger les effets de granulométrie et minéralogie.

Etant donné la faible variation de la composante principale de la matrice des végétaux, nous avons testé une méthode de préparation à masse constante. Les essais se sont portés sur plusieurs masses comprises entre 100 et $2000 \mathrm{mg}$. Les mesures à partir des cellules dont li masse d'échantillon est supérieure à $250 \mathrm{mg}$ montrent que les résultats obtenus sont équivalents en terme de concentration déduite, et ce pour l'ensemble des éléments d'intérêt. Dès lors, nous 
avons choisi de travailler avec une masse de $300 \mathrm{mg}$ qui constituent un bon compromis entre une bonue couverture de la surface analytique et une préparation de plusieurs cellules en vue des tests de reproductibilité compte tenu de la quantité des végétaux disponibles. Ceci permet de réduire considérablement la consommation en poudre végétale et donc d'améliorer les performances d'analyse de la fraction minérale dans les végétaux par rapport à une préparation par pastillage.

Une vingtaine de végétaux de références $\left(\mathrm{NIST}^{9}, \mathrm{IPE}^{10}\right.$ et $\mathrm{BCR}^{11}$ ) ont servi à étalonner l'analyse des végétaux par la procédure développée [13]. Un exemple de l'étalonnage effectué est illustré par la Figure 1.

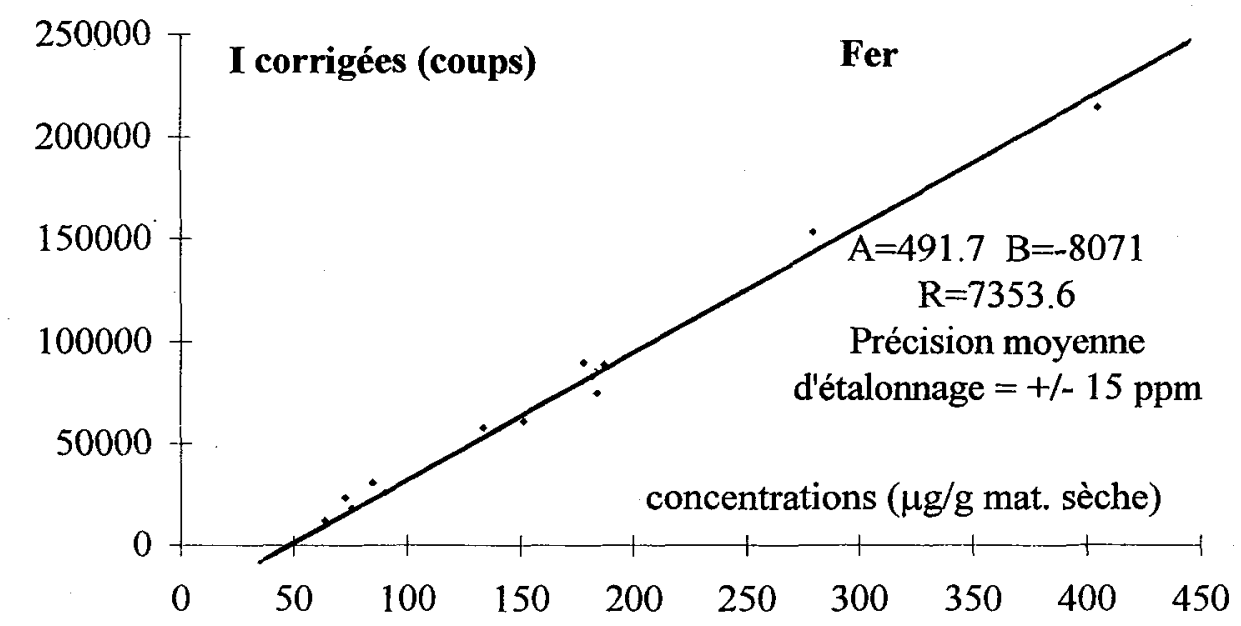

Figure 1: Courbe d'étalonnage pour l'analyse du fer (Fe $\mathrm{K}_{\alpha 1}$ )par SFX sur des poudres végétales mises en cellule

La teneur en silice des herbes pouvant être élevée selon les observations écologiques, nous avons mené l'analyse du silicium avec une préparation de type perles, décrite dans la partie concernant la procédure d'analyse des cendres.

\section{PROCEDURE D'ANALYSE DES CENDRES :}

2.1. Caractéristiques des cendres analysées :

Afin de contrôler in-situ le type de feu simulé, nous avons systématiquement mesuré le rapport $\mathrm{CO} / \mathrm{CO}_{2}$ des émissions, considéré comme le traceur expérimental de la qualité de combustion [4].

Nous avons réalisé au moins deux reconstitutions de chacun des processus "purs" de combustion ("flaming" ou "smoldering") avec un combustible "pur" (Loudetia mort ou SAFARI) afin d'étudier d'une part la reproductibilité de nos reconstitutions, et d'autre part la spécificité des produits formés. Pour chaque expérience, les cendres obtenues ont été soigneusement séparées des résidus imbrûlés et pesées pour quantifier, par ailleurs, l'efficacité du feu reconstitué.

\footnotetext{
${ }^{9}$ NIST : National Institute of Standards

${ }^{10}$ IPE : International Plants Exchange

it BCR : Bureau Communautaire de Références
} 


\subsection{Mise en oeuvre des analyses :}

L'analyse quantitative des constituants minéraux d'un tel échantillon est possible par SFX, à la condition de prendre en compte les effets de matrice perturbant la relation "Signal Mesuré-Concentration". Afin de minimiser ces effets, nous avons préparé, à partir de nos cendres, des échantillons sous forme de filtres, considérés en SFX comme des échantillons en couche mince pour lesquels l'intensité mesurée de l'élément considéré est directement proportionnelle à sa masse sur le filtre et donc à sa concentration dans l'échantillon initial $[16,17]$. Ainsi, pour déterminer les éléments majeurs et mineurs de nos cendres, nous avous mis ces dernières en suspension dans un liquide vecteur chimiquement inerte vis-à-vis des composants minéraux à analyser. Pour cela, l'échantillon est au préalable finement broyé (granulométrie $<5 \mu \mathrm{m}$ ) afin de satisfaire aux exigences granulométriques indispensables à une analyse quantitative menée en SFX sur un échantillon initialement en poudre [16]. Une partie aliquote est alors mise dans du cyclohexane, liquide vecteur généralement choisi pour sa neutralité chimique vis-à-vis des constituants minéraux [16]. La solution obtenue est ensuite agitée et ultrasonnée quelques secondes pour homogénéisation, puis transférée sur une membrane filtrante à l'aide d'un filtrateur à liquide dont la géométrie du filtre obtenu est parfaitement adaptée à celle analysée par le spectromètre employé. Les membranes utilisées sont en polycarbonate avec la taille des pores calibrée à $0,4 \mu \mathrm{m}$, montées sur support de tension Fil-Clip [18].

L'hypothèse de travail étant la stricte conservation du bilan matière lors du transfert sur la membrane, des tests de vérification de la nəutralité chimique du cyclohexane vis-à-vis de nos éléments d'intérêt ont été effectués. Pour cela, nous avons récupéré le cyclohexane ayant servi à la mise en suspension d'un échantillon après son passage à travers la membrane filtrante. Une dizaine de gouttes de celui-ci, après préconcentration par évaporation, a été alors déposée sur une membrane vierge du même type que celle utilisée pour la filtration des cendres mises en suspension. L'échantillon ainsi préparé est ensuite séché dans une atmosphère propre à la température ambiante pour faire évaporer le cyclohexane et déposer les sels que celui-ci aurait pu solubiliser lors de la mise en suspension. Les membranes ainsi fabriquées, se présentent comme des filtres, et sont donc analysables par SFX selon la même méthode de "couchesminces". Les résultats des analyses n'ayant jamais montré de quantités en éléments d'intérêt qui soit significativement différentes de celles des membranes vierges, notre hypothèse de travail a donc été validée. Ainsi, la conservation des cendres que nous avons transférées sur les membranes a été parfaitement respectée. Les filtres-échantillons peuvent alors être analysés pour déterminer les masses $\mathbf{M}_{\mathbf{i}}$ des différents éléments " $i$ ". Par cette méthode, les seuils de détection analytiques sont généralement de l'ordre de quelques nannogrammes par élément et par filtre. Si on rapporte à une masse moyenne filtrée de l'ordre du milligramme, cette valeur correspond à une limite de détection en teneur relative de quelques ppm. Le procédé utilisé est donc particulièrement sensible, et comparable en performance à l'analyse qui serait obtenue par nombre d'autres méthodes. De plus, elle présente l'avantage spécifique d'être conservative. II est ainsi possible d'archiver les filtres-échantillons et/ou de les analyser si nécessaire par une autre méthode complémentaire. La précision moyenne des analyses de filtres par SFX pour nos éléments d'intérêt est généralement meilleure que $5 \%$ en relatif Son amélioration éventuelle n'est en fait pas limitée par le procédé que nous avons mis en place, mais par la difficulté de fabriquer les filtres-étalons avec une meilleure précision [17].

A partir des masses sur filtres $M_{i}$, nous pouvons déduire les concentrations $C_{i}$ des différents éléments " $\mathrm{i}$ " contenus dans les cendres initiales suivant la relation :

$$
\mathbf{C}_{\mathrm{i}}=\mathbf{M}_{\mathbf{i}} / \mathbf{M}_{\text {totale }}
$$


avec $M_{\text {totale }}$ : la masse totale des cendres transférée sur filtre. Cependant, celle-ci n'est pas quantifiable précisément à l'heure actuelle avec une analyse globale des filtres, même si nous pouvons estimer qu'elle est de l'ordre du milligramme. Afin de déterminer cette masse, nous avons recherché un élément " $\mathrm{j}$ " contenu dans les cendres, dont la concentration $\mathrm{C}_{\mathrm{j}}$ peut être directement déterminée par SFX au moyen d'un autre protocole de préparation de l'échantillon. Comme $\mathrm{Mj}$ est connu grâce à l'analyse des filtres, $\mathrm{M}_{\text {totale }}$ peut alors être facilement calculable à l'aide de la relation (I). Dans ce but, nous avons opté pour une préparation d'échantillon sous forme de solutions solides vitrifiées, une méthode très couramment utilisée en SFX et qui permet également de supprimer des effets perturbateurs liés aux effets de matrice [16]. Pour cela, $0,1 \mathrm{~g}$ de cendres sont dilués dans $9,9 \mathrm{~g}$ de fondant (tétraborate de lithium : $\mathrm{Li}_{2} \mathrm{~B}_{4} \mathrm{O}_{7}$ ). Le mélange est ensuite mis dans un four dont la température a été portée à $1050^{\circ} \mathrm{C}$. Le milieu fondu formé est homogénéisé par agitation puis coulé dans un moule dont la forme et l'état de surface permettent d'obtenir, après refroidissement, un verre solide de géométrie parfaitement adaptée à l'analyse avec le spectromètre employé. Avec le taux de dilution choisi $(1 / 100)$ et le fondant utilisé $\left(\mathrm{Li}_{2} \mathrm{~B}_{4} \mathrm{O}_{7}\right)$, les échantillons ainsi préparés présentent des effets de matrice négligeables, la relation entre le signal de fluorescence $\mathrm{X}$ de l'élément mesuré et sa concentration correspondante est donc de stricte proportionnalité [16]. Cependant, il faut noter que la température de fusion utilisée pourrait entraîner une volatilisation et donc une perte éventuelle de certains composés de l'échantillon $\left(\mathrm{CaCO}_{3}, \mathrm{KCl}, \ldots\right)$. Nous avons donc utilisé cette préparation et analysé les échantillons solides obtenus vis-à-vis du seul élément pour lequel nous avons la certitude d'une parfaite conservation lorsqu'il est porté à une température de $1050^{\circ} \mathrm{C}:$ le silicium qui, à priori, n'est présent que sous forme de silice puisque les cendres initiales ont été diluées dans un fondant fortement oxydant [16].

Pour l'analyse du silicium par le spectromètre utilisé, les conditions instrumentales ont été les suivantes : un tube à anode de rhodium sous $35 \mathrm{kV}$ et $75 \mathrm{~mA}$, un cristal de Pentaérytritol (PET) de distance réticulaire $0,874 \mathrm{~nm}$ couplé à un détecteur de type compteur proportionnel à flux gazeux $\mathrm{Ar}-\mathrm{CH}_{4}(\mathrm{CPFG})$ et un temps de comptage de 60 secondes.

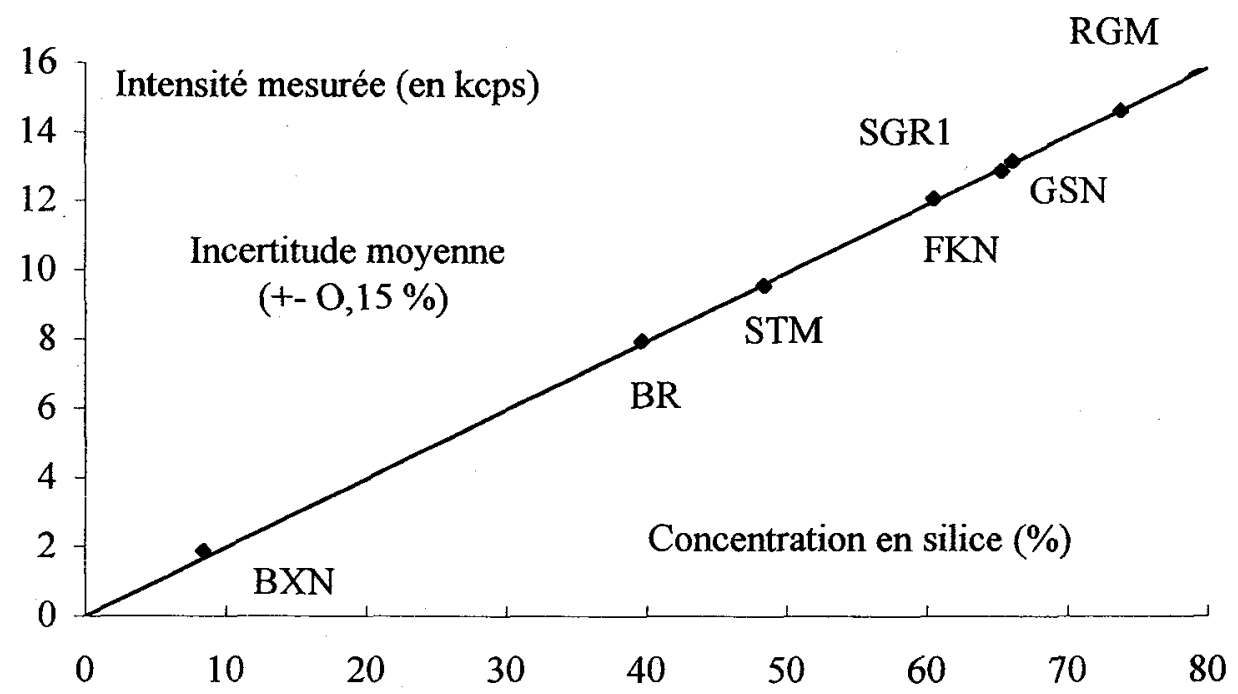

Figure 2: Courbe d'étalonnage pour l'analyse de la silice par SFX sur des solutions solides vitrifiées 
Afin d'étalonner l'intensité mesurée (Figure 2), de la raie $K \alpha_{1,2}$ du silicium $(0,7127 \mathrm{~nm}$ soit un angle goniométrique de Bragg de $2 \theta=109^{\circ} 22$ ), nous avons préparés des échantillons solides étalons à partir des matériaux de référence internationaux de type géostandard, fournis par le Centre de Recherche Pétrographique et Géologique (CRPG) et l'Association Nationale de la Recherche Technique (ANRT) de Vandoeuvre-les-Nancy [19].

Avec les conditions instrumentales utilisées, le seuil de détection analytique est de $1 \%$ avec une erreur statistique, liée à la fois à l'étalonnage et aux erreurs de mesures de $\pm 0,15 \%$ en absolu, sur la gamme de concentration allant de 8 à $75 \%$ en silice.

Les concentrations en silicium des cendres des différentes combustions reconstituées peuvent alors être directement obtenues par ce protocole analytique. Les valeurs ainsi déterminées serviront ensuite à calculer les différentes masses totales de cendres déposées sur filtre selon le protocole précédent de préparation.

\section{RESULTATS-DISCUSSION :}

Les résultats obtenus pour les végétaux sont reportés dans le Tableau 1. Le seuil de détection des différents élements sont de l'ordre de $10 \mathrm{ppm}$ et la précision moyenne analytique est en général meilleur que $10 \%$ en relatif [13]. Les valeurs observées pour le potassium et le phosphore sont en bon accord avec les données de la littérature mentionnées pour les mêmes herbes. Elles montrent également que l'ensemble des éléments recherchés permet de caractériser la quasi-intégralité de la matrice végétale : que ce soit pour Loudetia ou SAFARI, le bilan des éléments analysés représente plus de $97 \%$ de la masse totale du végétal.

\begin{tabular}{|c|c|c|c|c|c|c|c|c|c|}
\hline & C (\%) & H (\%) & O(\%) & \multicolumn{2}{|c|}{$\mathbf{N}(\%)$} & $\mathrm{Fe}(\%)$ & $\operatorname{Mn}(\%)$ & 6) $\mathrm{Ca}(\%)$ & K (\%) \\
\hline Loudetia & 40,44 & 5,33 & 42,66 & \multicolumn{2}{|c|}{0,26} & 0,0322 & 0,0360 & 0,519 & 0,349 \\
\hline \pm & 0,46 & 0,07 & 1,66 & \multicolumn{2}{|c|}{0,02} & 0,0004 & 0,0005 & & \\
\hline SAFARI & 42,57 & 5,72 & 46,27 & \multicolumn{2}{|c|}{0,29} & 0,0253 & 0,0109 & & \\
\hline \multirow[t]{2}{*}{ \pm} & 0,08 & 0,02 & 0,00 & \multicolumn{2}{|c|}{0,03} & 0,0008 & \multirow{2}{*}{$\frac{0,0003}{\mathrm{Va}(\%)}$} & 0,011 & \multirow{2}{*}{$\begin{array}{c}0,006 \\
\text { Total(\%) }\end{array}$} \\
\hline & $\mathrm{Cl}(\%)$ & $\mathbf{S}(\%)$ & & & & $(\%)$ & & SiO2(\%) & \\
\hline Loudetia & 0,099 & & & & & & 0,02 & & 97,21 \\
\hline \pm & 0,003 & 0,000 & & & & & 0,001 & 0,250 & 2,48 \\
\hline SAFARI & 0,129 & 0,080 & & & & & 0,057 & & 100,47 \\
\hline \pm & 0,002 & 0,002 & 0,0 & & & 04 & 0,006 & 0,250 & 0,42 \\
\hline
\end{tabular}

Tableau 1: Composition chimique de Loudetia mort et de SAFARI

Les compositions globale et minérale des cendres sont illustrées par les Figures 3 et 4 . Les résultats obtenus soulignent que cette composition dépend du mode de combustion (Figures 2 et 3 ). De plus, quel que soit le combustible utilisé, ils indiquent que les cendres formées sont riches en silicium sous forme de silice. L'ensemble des éléments analysés représente la composition quasi-totale de la matrice des cendres [20].

Par ailleurs, nous notons que la composition minérale des cendres est étroitement liée à celle des végétaux combustibles. En effet, à l'exception de $\mathrm{Fe}, \mathrm{K}$ et $\mathrm{Ca}$, le profil minéral des cendres est identique à celui des herbes qui leur ont donné naissance (Figures 4 et 5). 


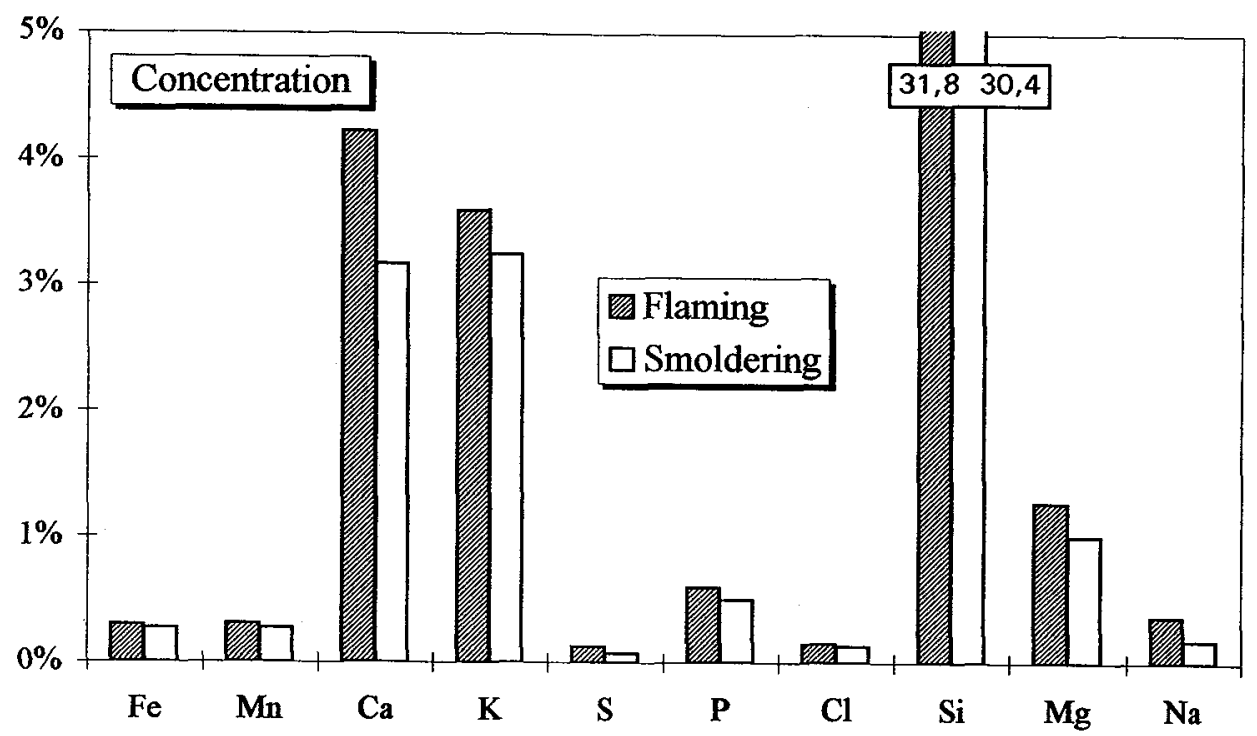

Figure 3: Composition minérale des cendres en fonction des modes de combustion avec Loudetia mort comme combustible

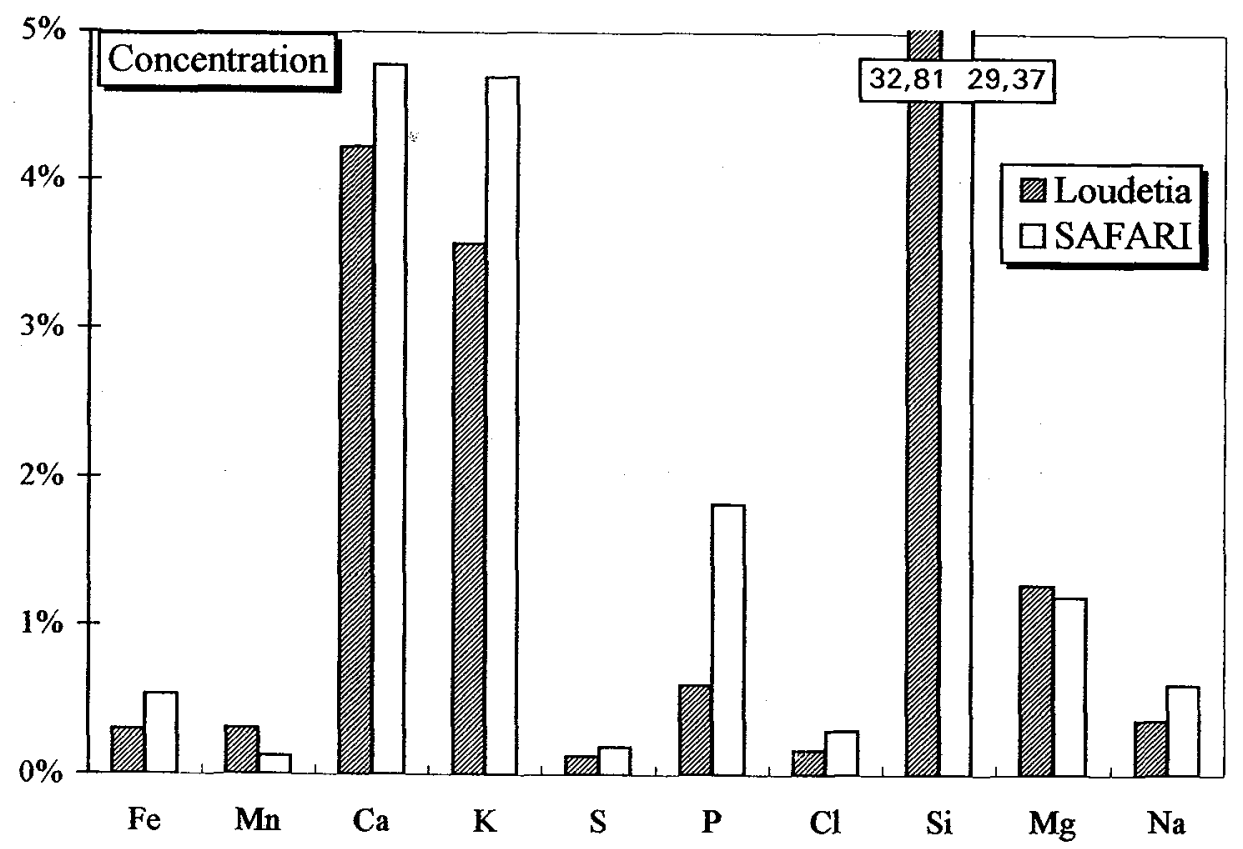

Figure 4: Composition minérale des cenảres en fonction des combustibles pour ie mode "flaming" 


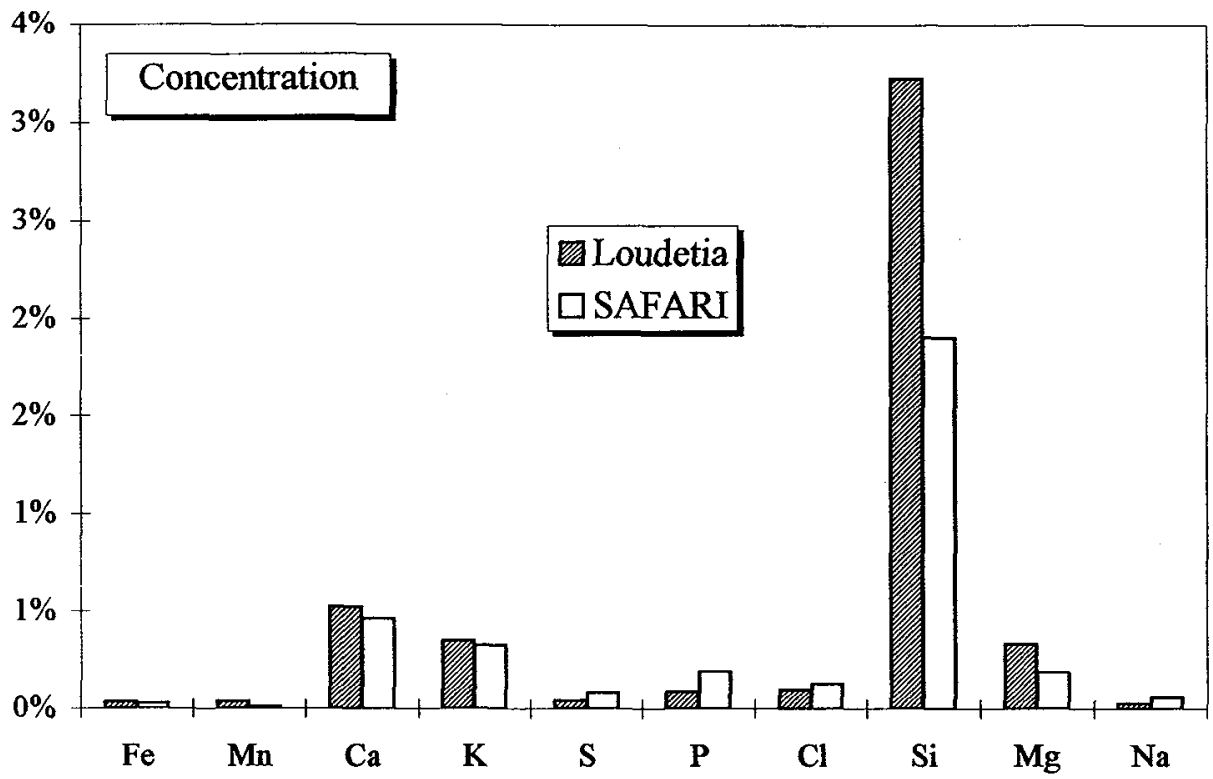

Figure 5: Composition minérale des végétaux combustibles

\section{CONCLUSION :}

Les différentes procédures développées pour l'analyse dẹs végétaux combustibles et des cendres formées par SFX ont permis de documenter pour la première fois la quasiintégralité de la composition minérale de ces échantillons. Les résultats obtenus montrent l'importance des facteurs d'influence (modes de combustion et composition des combustibles) sur la formation des cendres, et ce avec la même qualité analytique que pour les autres fractions produites (gaz et particules). Le bon accord des données issues des différentes procédures de préparation d'échantillon montre que ces dernières sont effectivement complémentaires. Ceci permet de souligner le potentiel de la SFX pour l'analyse de ces échantillons jusqu'à présent peu abordés par cette technique.

\section{Références}

[1] Crutzen P.J., Andreae M.O., Science, 250 (1990) 1669-1678.

[2] Andreae M.O., In "Global Biomass Burning : Atmospheric, Climatic and Biospheric Implications" (Levine J.S. ed, MIT Press, 1991) p. 3-21.

[3] Gaudichet A., Echalar F., Chatenet B., Quisefit J.P., Malingre G., Cachier H., BuatMénard P., Artaxo P., Maenhaut W., J.Atmos. Chem., 22 (1995) 19-39. 
[4] Garivait S., Approche physico-chimique de la formation des composés produits par les feux de savane - Développement d'un modèle dynamique basé sur les lois d'équilibres thermochimiques (Thèse, Université Paris 7, 1995).

[5] Ward D.E., In "Fires in tropical Biota", (Goldammer J.G. ed, Springer-Verlag, 1990) p. 418-436.

[6] Menaut J.C., Sécheresse, 4 (1995) 251-264.

[7] Villecourt P., Schmidt W., César J., Rev. Ecol. Biol. Sol, 16 (1979) 9-15.

[8] Pinta M., Le Comité Inter-Instituts d'Etude des Techniques de Diagnostic Foliaire (CII), Analusis, 3 (1975) 345-353.

[9] Hoenig M., Van Hoeyweghen P., Liboten J., Analusis, 7 (1979) 104-108.

[10] Ablgrén M., Kontkanen A., Vattulainen K, Vekvilänen H., Analyst, 113 (1988) 285290.

[11] N. Wieberneit, F.B. Meyberg, W. Dannecker, In "CANAS'93 : Colloquim Analytische Atomspektroskopie" (1993) p. 767.

[12] R. Oliver, Analusis, 7, (1979) 38-45.

[13] Garivait S., Quisefit J.P., De Chateaubourg P., Malingre G., X-Rays Spectrom., (sous presse).

[14] K. Norrish, J.T. Hutton, X-Ray Spectrom., 6, (1977a) 6-11.

[15] K. Norrish, J.T. Hutton, X-Ray Spectrom., 6, (1977b) 11-17.

[16] R; Tertian, F. Claisse, Principles of Quantitative X-Ray Fluorescence Analysis, Heyden, London (1982).

[17] Losno R., Bergametti G. et Mouvier G., Environ. Technology Letter, 8 (1987) 77-82.

[18] Prigent H. et Quisefit J.P., Brevet BF 8906801 (1991).

[19] Govindaraju K, Geostandard Newsletter, XVII (1994) 1-158.

[20] Garivait S., Quisefit J.P., Analusis (sous presse). 